\title{
COMBINING ABILITY OF SOME BREAD WHEAT GENOTYPES USING LINE X TESTER ANALYSIS UNDER THE NEW VALLEY CONDITIONS
}

\author{
MOUBARAK, M. Y. GH. AND HODA, M. M. EL-GHARBAWY
}

Wheat Research Department, Field Crops Research Institute, ARC., Giza Egypt.

(Manuscript received 29 November 2011)

\begin{abstract}
Experiments of this investigation were carried out at the experimental farm of the New Valley Agricultural Research Station, Agricultural Research Center, Egypt, during 2009/2010 and 2010/2011 seasons. The objective was to estimate the general (GCA) and specific combining abilities (SCA) of twelve genotypes for some quantitative characters in bread wheat using line $x$ tester analysis. Twelve bread wheat genotypes were crossed to three local wheat cultivars (Sakha-93, Gemmeiza-9 and Sids-12) to produce thirty six test crosses. The studied characters were plant height, spikes per plant, kernels per spike, spike kernel weight, 100-kernel weight and grain yield per plant. Results indicated that, the genotypes (parents and crosses) exhibited significant variance for all studied characters, indicating the presence of genotypic differences. Mean squares due to parents vs. crosses were significant for all studied characters, except for spike kernel weight. The GCA/SCA ratio did not exceed unity for all studied characters, indicating that SCA variance was more important than GCA variance and thus non-additive genetic variance was the predominant component that controls the inheritance of all studied characters. Results indicated that Sids-12 could be recommended as a parental genotype for wheat breeding programs. The lines number two and nine had The highest GCA effects were shown by line 2 for spikes per plant, kernels per spike, line $\mathrm{g}$ for spike kernel weight and grain yield per plant. The lines and testers which showed higher combining ability effects for grain yield were also good combiners for at least one of the three yield components.
\end{abstract}

Key words: Wheat, line $x$ tester, combining ability, type of gene action.

\section{INTRODUCTION}

The common bread wheat (Triticum aestivum, L.) is one of the major cereal crops which is widely grown not only in Egypt but also throughout the world as a prime food cereal. Increasing wheat production to narrow the gap between production and consumption is considered one of the main goals of wheat breeders in Egypt as well as in most countries all over the world. Combining ability analysis has been used extensively in cross pollinated crops to classify the parental lines in terms of their ability to be combine in hybrids. In self pollinated crops like wheat, combining ability analysis could also be useful to determine the relative magnitude of additive 
and non additive type of gene actions in the expression of a character of interest. Therefore, studying combining ability in wheat will be helpful in choosing parents in a hybridization program and the suitable breeding procedure to improve the trait of interest.

General and specific combining ability estimates have been calculated by several wheat workers (Abd El-Rahman, 1991). These studies in general, indicated that the large part of the total genetic variation for yield was due to general combining ability variance which measures additive genetic variance. On the other hand, specific combining ability variance indicates non-additive genetic variance.

The line $x$ tester analysis was used to estimate both general and specific combining ability variances and effects for yield and its components in wheat by several investigators such as Srivastava and Dhirendra (2008), Akbar et. al. (2009) and Krystkowiak et. al. (2009). Most studies on wheat revealed that general combining ability (GCA) was more important than specific combining ability (SCA) for spikes per plant (Eissa 1993). On the other hand, GCA to SCA variance ratio revealed the predomination of non-additive gene action for plant height, spikes per plant, kernel weight and grain yield per plant (Akbar et. al. , 2009). However, additive (GCA) and non-additive (SCA), genetic effects were observed for grain yield per plant, kernels per spike, kernel weight and spikes per plant (Chowdhry et. al. 1996). On the other hand, El-Beially and El-sayed (2002) concluded that mean squares due to GCA and SCA were significant for plant height, spikes per plant, kernels per spike, kernel weight and grain yield per plant.

The objective of this study was to estimate general and specific combining ability variances and effect of twelve genotypes using line $x$ tester analysis for the most important economic characters of wheat, i.e., plant height, spikes per plant, kernels per spike, spike kernel weight, kernel weight and grain yield per plant under the New Valley conditions in twelve wheat genotypes.

\section{MATERIALS AND METHODS}

The present study was carried out at the New Valley Agricultural Research Station, during the two successive growing seasons, 2009/2010 and 2010/2011. In 2009/2010 season, twelve bread wheat genotypes were crossed with three local wheat cultivars (Sakha-93, Gemmeiza-9 and Sids-12) as testers "T" to produce thirty six test crosses. The pedigree of the parental genotypes is presented in Table (1).

In 2010/2011 growing season, the thirty six $F_{1}$ crosses and their parental genotypes were evaluated for grain yield and its contributing characters using a 
randomized completed block design with four replications. Each experimental plot consisted of three rows; three meters long and $20 \mathrm{~cm}$ apart and seeds were spaced at $10 \mathrm{~cm}$ within rows for each studied genotype. The recommended cultural practices were applied at the proper time. Data were recorded on ten individual guarded plants from each plot for plant height, spikes per plant, kernels per spike, spike kernels weight, 100-kernel weight and grain yield per plant.

The obtained data were subjected to line $\mathrm{x}$ tester analysis as outlined by Kempthorne (1957).

Table 1. Name and pedigree of the parental genotypes.

\begin{tabular}{|l|l|l|}
\hline \multicolumn{1}{|c|}{ Line No. $^{|c|}$ Line name and pedigree } & \multicolumn{1}{c|}{ Origin } \\
\hline $\mathrm{L}_{1}$ & Sids-7 / Sahel-1 & Egypt \\
\hline $\mathrm{L}_{2}$ & XINCHUN6-OCHN & Mexico \\
\hline $\mathrm{L}_{3}$ & Maya "S" /3/F 35-70/Mo//Nac/Giza-160//Debera & Egypt \\
\hline $\mathrm{L}_{4}$ & Giza-168=(MIL/BUC//Seri) & Egypt \\
\hline $\mathrm{L}_{5}$ & Maya "S" /3/F 35-70/Mo//Nac/Giza-160// Sids-1 & Egypt \\
\hline $\mathrm{L}_{6}$ & Sids-7=(Maya " S" / Mon " S" // CMH 74 A. 592/3/ Sakha 8*2) & Egypt \\
\hline $\mathrm{L}_{7}$ & Debera / Giza-164 & Egypt \\
\hline $\mathrm{L}_{8}$ & Maya "S" /3/F 35-70/Mo//Nac/Giza-160 & Egypt \\
\hline $\mathrm{L}_{9}$ & Sahel-1=(Nc 732 / P1MA / Veery " S") & Egypt \\
\hline $\mathrm{L}_{10}$ & Sids-7 / Giza-164 & Egypt \\
\hline $\mathrm{L}_{11}$ & Sids-7 / Sids-1 & Egypt \\
\hline $\mathrm{L}_{12}$ & Sahel-1 / Debera & Egypt \\
\hline $\mathrm{T}_{2}$ ster No. & & \\
\hline $\mathrm{T}_{1}$ & Sakha-93 & Egypt \\
\hline $\mathrm{T}_{2}$ & Gemmeiza-9 & Egypt \\
\hline $\mathrm{T}_{3}$ & Sids-12 & Egypt \\
\hline
\end{tabular}

\section{RESULTS AND DISCUSSION}

The mean performance of wheat genotypes (15 parents and 36 crosses) for all studied characters is presented in Table (2). The analysis of variance for all studied characters is presented in Table (3). Genotypes exhibited significant variation ( $P \geq$ 0.05) for all studied characters, indicating the presence of genetic diversity among these fifty genotypes. Mean squares due to parents vs. crosses as an indication to average heterosis across all crosses were significant for all characters, except for spike kernel weight which did not reach the significance level. Furthermore, crosses mean squares of line $x$ tester analysis indicated that differences due to all lines and testers 
were significant for all characters. The GCA/SCA ratios did not exceed unity for all studied characters, indicating that SCA variances were more important than GCA variances and thus non-additive genetic variance was the predominant component controlling the inheritance of all studied characters. Similar results were previously reported by Koumber (2007) and Akbar et. al. (2009).

\section{General combining ability (GCA)}

Estimation of GCA for the twelve lines and the three testers are presented in Table (4). Positive values would be of interest in all yield characters under study. On the contrary, for plant height, negative values would be useful from the breeder's point of view. For plant height, the five wheat lines 1, 3, 4, 6 and 10 in addition to the tester Sakha-93 showed negative significant general combining ability effects, revealing that these parental lines may be taken in consideration when selecting for resistance to lodging. Reduced lodging has usually been observed to be associated with decrease in culms length and consequently, the search for suitable genetic variation and the incorporation of sources of short stiff straw into breeding program have received much attention. Similar results were obtained by Moubarak (2003), Koumber (2007) and Srivastava Dhirendra (2008).

Table 2. Mean performance of the line $x$ tester cross and parental genotypes for the studied characters (2010/2011).

\begin{tabular}{|c|c|c|c|c|c|c|c|}
\hline \multicolumn{2}{|c|}{ Hybrids } & $\begin{array}{l}\text { Plant } \\
\text { height } \\
(\mathrm{cm} .)\end{array}$ & $\begin{array}{l}\text { spikes/ } \\
\text { plant }\end{array}$ & $\begin{array}{l}\text { kernels/ } \\
\text { spike }\end{array}$ & $\begin{array}{l}\text { Spike } \\
\text { kernel } \\
\text { weight (g) }\end{array}$ & $\begin{array}{l}100- \\
\text { kernel } \\
\text { weight } \\
\text { (g) }\end{array}$ & $\begin{array}{l}\text { Grain yield } \\
\text { / plant (g) }\end{array}$ \\
\hline \multirow{3}{*}{$\mathrm{L}_{1}$} & $\times \mathrm{T}_{1}$ & 82.5 & 6.0 & 46.25 & 3.20 & 5.07 & 23.17 \\
\hline & $\times T_{2}$ & 90.0 & 5.5 & 40.25 & 1.81 & 4.64 & 11.32 \\
\hline & $\times T_{3}$ & 90.0 & 4.5 & 51.25 & 2.65 & 4.62 & 16.33 \\
\hline \multirow{3}{*}{$\mathrm{L}_{2}$} & $\times \mathrm{T}_{1}$ & 90.0 & 5.5 & 58.25 & 3.22 & 5.21 & 19.52 \\
\hline & $\mathrm{xT}_{2}$ & 95.0 & 6.0 & 60.50 & 2.97 & 4.40 & 17.91 \\
\hline & $\times T_{3}$ & 100.0 & 7.5 & 67.00 & 3.61 & 5.45 & 26.63 \\
\hline \multirow{3}{*}{$\mathrm{L}_{3}$} & $\mathrm{xT}_{1}$ & 85.0 & 5.5 & 50.00 & 3.09 & 4.87 & 18.53 \\
\hline & $\times T_{2}$ & 85.0 & 6.0 & 63.75 & 2.19 & 4.81 & 11.83 \\
\hline & $\times T_{3}$ & 95.0 & 7.5 & 57.00 & 2.65 & 4.49 & 15.62 \\
\hline \multirow{3}{*}{$L_{4}$} & $\times T_{1}$ & 80.0 & 5.5 & 53.50 & 2.43 & 4.66 & 14.23 \\
\hline & $\times T_{2}$ & 95.0 & 4.5 & 64.00 & 2.54 & 3.94 & 12.46 \\
\hline & $\times T_{3}$ & 80.0 & 4.0 & 55.50 & 2.61 & 4.43 & 11.52 \\
\hline \multirow{3}{*}{$\mathrm{L}_{5}$} & $\times T_{1}$ & 90.0 & 6.5 & 53.50 & 2.57 & 4.71 & 24.60 \\
\hline & $\times T_{2}$ & 90.0 & 5.5 & 54.50 & 2.61 & 3.97 & 22.78 \\
\hline & $x T_{3}$ & 100.0 & 5.5 & 46.75 & 2.71 & 4.70 & 13.40 \\
\hline \multirow{3}{*}{$\mathrm{L}_{6}$} & $x T_{1}$ & 77.5 & 4.5 & 38.50 & 2.92 & 5.07 & 15.04 \\
\hline & $\times T_{2}$ & 100.0 & 6.5 & 60.00 & 2.42 & 4.75 & 20.23 \\
\hline & $\times T_{3}$ & 85.0 & 3.5 & 79.50 & 3.95 & 5.28 & 13.04 \\
\hline $\mathrm{L}_{7}$ & $x \mathrm{~T}_{1}$ & 95.0 & 4.5 & 48.75 & 2.94 & 4.61 & 10.52 \\
\hline
\end{tabular}




\begin{tabular}{|c|c|c|c|c|c|c|c|}
\hline & $\times T_{2}$ & 95.0 & 6.0 & 59.75 & 2.65 & 4.18 & 18.95 \\
\hline & $\times T_{3}$ & 100.0 & 6.5 & 77.75 & 3.52 & 4.62 & 24.59 \\
\hline \multirow{3}{*}{$\mathrm{L}_{8}$} & $\times T_{1}$ & 90.0 & 6.0 & 39.50 & 1.82 & 4.56 & 10.51 \\
\hline & $\times T_{2}$ & 90.0 & 6.5 & 53.50 & 2.46 & 4.24 & 14.00 \\
\hline & $\times T_{3}$ & 105.0 & 6.0 & 59.25 & 3.52 & 5.03 & 21.51 \\
\hline \multirow{3}{*}{$\mathrm{L}_{9}$} & $\mathrm{xT}_{1}$ & 100.0 & 7.5 & 65.50 & 3.02 & 4.94 & 19.46 \\
\hline & $\times T_{2}$ & 90.0 & 6.5 & 65.25 & 2.99 & 3.99 & 22.75 \\
\hline & $\times T_{3}$ & 90.0 & 6.5 & 64.50 & 3.26 & 4.60 & 18.42 \\
\hline \multirow{3}{*}{$\mathrm{L}_{10}$} & $\times T_{1}$ & 75.0 & 5.5 & 47.75 & 2.41 & 4.85 & 15.99 \\
\hline & $\times T_{2}$ & 90.0 & 6.5 & 62.00 & 2.63 & 4.66 & 14.99 \\
\hline & $\times T_{3}$ & 95.0 & 5.5 & 50.75 & 2.71 & 4.95 & 16.38 \\
\hline \multirow{3}{*}{$\mathrm{L}_{11}$} & $\times \mathrm{T}_{1}$ & 80.0 & 5.0 & 57.50 & 2.75 & 5.09 & 12.37 \\
\hline & $\times T_{2}$ & 85.0 & 5.5 & 48.00 & 2.59 & 4.58 & 13.55 \\
\hline & $\times T_{3}$ & 110.0 & 7.5 & 52.75 & 2.68 & 5.09 & 16.23 \\
\hline \multirow{3}{*}{$\mathrm{L}_{12}$} & $\times \mathrm{T}_{1}$ & 85.0 & 5.5 & 50.75 & 2.93 & 4.98 & 18.45 \\
\hline & $\times T_{2}$ & 97.5 & 7.0 & 67.00 & 2.65 & 4.58 & 20.47 \\
\hline & $\times T_{3}$ & 95.0 & 6.5 & 69.25 & 3.23 & 4.76 & 17.84 \\
\hline \multicolumn{2}{|l|}{ LSD 0.05} & 1.178 & 0.791 & 4.397 & 0.281 & 0.377 & 1.247 \\
\hline \multicolumn{8}{|c|}{ Lines } \\
\hline \multicolumn{2}{|l|}{$\mathrm{L}_{1}$} & 92.5 & 7.5 & 63.50 & 2.54 & 4.50 & 20.97 \\
\hline \multicolumn{2}{|l|}{$\mathrm{L}_{2}$} & 105.0 & 5.5 & 45.25 & 3.04 & 5.66 & 15.10 \\
\hline \multicolumn{2}{|l|}{$\mathrm{L}_{3}$} & 90.0 & 8.5 & 50.50 & 3.10 & 4.35 & 23.54 \\
\hline \multicolumn{2}{|l|}{$\mathrm{L}_{4}$} & 85.0 & 4.5 & 56.00 & 2.31 & 4.23 & 10.01 \\
\hline \multicolumn{2}{|l|}{$\mathrm{L}_{5}$} & 105.0 & 9.0 & 67.00 & 2.71 & 4.16 & 22.14 \\
\hline \multicolumn{2}{|l|}{$\mathrm{L}_{6}$} & 75.0 & 3.0 & 54.75 & 2.75 & 5.21 & 9.09 \\
\hline \multicolumn{2}{|l|}{$\mathrm{L}_{7}$} & 115.0 & 6.0 & 76.00 & 2.79 & 3.53 & 18.17 \\
\hline \multicolumn{2}{|l|}{$\mathrm{L}_{8}$} & 95.0 & 9.0 & 54.00 & 2.25 & 4.30 & 21.47 \\
\hline \multicolumn{2}{|l|}{$\mathrm{L}_{9}$} & 97.5 & 4.5 & 60.25 & 2.38 & 3.84 & 11.99 \\
\hline \multicolumn{2}{|l|}{$\mathrm{L}_{10}$} & 90.0 & 5.5 & 63.00 & 2.86 & 4.56 & 13.80 \\
\hline \multicolumn{2}{|l|}{$\mathrm{L}_{11}$} & 105.0 & 9.5 & 52.00 & 2.70 & 5.20 & 23.06 \\
\hline \multicolumn{2}{|l|}{$\mathrm{L}_{12}$} & 100.0 & 7.5 & 55.25 & 2.77 & 4.70 & 20.65 \\
\hline \multicolumn{2}{|l|}{ LDS 0.05} & 1.621 & 0.688 & 3.701 & 0.238 & 0.294 & 1.408 \\
\hline \multicolumn{8}{|c|}{ Testers } \\
\hline \multicolumn{2}{|l|}{$\mathrm{T}_{1}$} & 75.0 & 6.5 & 51.00 & 2.46 & 4.75 & 18.07 \\
\hline \multicolumn{2}{|l|}{$\mathrm{T}_{2}$} & 95.0 & 5.5 & 60.00 & 3.56 & 4.06 & 15.35 \\
\hline \multicolumn{2}{|l|}{$\mathrm{T}_{3}$} & 95.0 & 6.5 & 76.25 & 3.61 & 4.83 & 23.40 \\
\hline \multicolumn{2}{|l|}{ LSD 0.05} & 5.768 & 0.816 & 5.183 & 0.374 & 0.493 & 1.725 \\
\hline
\end{tabular}


Table 3. Mean squares of the analysis of variance for the studied characters.

\begin{tabular}{|c|c|c|c|c|c|c|c|}
\hline \multirow{2}{*}{ Sources of variance } & \multirow{2}{*}{ d.f } & \multicolumn{6}{|c|}{ Mean squares } \\
\hline & & $\begin{array}{l}\text { Plant } \\
\text { height }\end{array}$ & $\begin{array}{l}\text { spikes/ } \\
\text { plant }\end{array}$ & $\begin{array}{l}\text { kernels/ } \\
\text { spike }\end{array}$ & $\begin{array}{l}\text { Spike } \\
\text { kernel } \\
\text { weight }\end{array}$ & $\begin{array}{l}\text { 100-kernel } \\
\text { weight }\end{array}$ & $\begin{array}{l}\text { Grain yield/ } \\
\text { plant }\end{array}$ \\
\hline $\begin{array}{l}\text { Replications } \\
\text { Genotypes } \\
\text { Parents } \\
\text { Crosses } \\
\text { Parents Vs. Crosses } \\
\text { Lines } \\
\text { Testers } \\
\text { Lines x testers } \\
\text { Error } \\
\text { GCA } \\
\text { SCA } \\
\text { GCA/SCA }\end{array}$ & $\begin{array}{c}3 \\
50 \\
14 \\
35 \\
1 \\
11 \\
2 \\
22 \\
150\end{array}$ & $\begin{array}{c}0.817 \\
320.373^{*} \\
481.667^{*} \\
249.107^{*} \\
556.567^{*} \\
178.977^{*} \\
1127.083^{*} \\
204.356^{*} \\
1.484 \\
0.528 \\
50.718 \\
0.010\end{array}$ & $\begin{array}{l}0.144 \\
7.226^{*} \\
14.552^{*} \\
3.875^{*} \\
21.949^{*} \\
4.937^{*} \\
1.861^{*} \\
3.528^{*} \\
0.304 \\
0.004 \\
0.806 \\
0.005\end{array}$ & $\begin{array}{c}18.319 \\
354.358^{*} \\
322.802^{*} \\
370.533^{*} \\
230.017^{*} \\
403.422^{*} \\
1317.299^{*} \\
268.018^{*} \\
8.819 \\
1.211 \\
64.800 \\
0.019\end{array}$ & $\begin{array}{l}0.011 \\
0.767^{*} \\
0.662^{*} \\
0.830^{*} \\
0.032 \\
0.825^{*} \\
3.661^{*} \\
0.575^{*} \\
0.041 \\
0.003 \\
0.134 \\
0.023\end{array}$ & $\begin{array}{l}0.128 \\
0.737^{*} \\
1.25^{*} \\
0.514^{*} \\
1.360^{*} \\
0.622^{*} \\
3.484^{*} \\
0.191^{*} \\
0.065 \\
0.004 \\
0.031 \\
0.122\end{array}$ & $\begin{array}{c}0.835 \\
80.928^{*} \\
97.786^{*} \\
75.905^{*} \\
20.721^{*} \\
86.470^{*} \\
10.544^{*} \\
76.565^{*} \\
0.817 \\
-0.008 \\
18.937 \\
-0.0004\end{array}$ \\
\hline
\end{tabular}

${ }^{*}$ Significant at 0.05 level of probability.

On the other hand, wheat lines number 2, 3, 8, 9, 12 and tester Gemmeiza-9 gave positive general combining ability effects for spikes per plant. Concerning kernels per spike, six parental lines $(2,4,6,7,9,12)$ and two testers (Gemmeiza-9 and Sids12) showed significant GCA effects proving to be good combiners in this respect. For spike kernel weight five wheat lines $(2,6,7,9$ and 12) and one tester (Sids-12) showed significant values of general combining ability effects. Wheat lines number 2 , $6,10,11$ and two testers (Sakha-93 and Sids-12) showed desirable general combining ability effects for kernel weight. For grain yield per plant, wheat lines 2, 5, 7, 9, 12 and tester Sids-12 were considered as good donors in this respect

Table 4. Estimates of general combining ability (GCA) effects for the studied characters.

\begin{tabular}{|l|l|l|l|l|l|l|}
\hline Lines & $\begin{array}{l}\text { Plant } \\
\text { height } \\
(\mathrm{cm} .)\end{array}$ & $\begin{array}{l}\text { spikes/ } \\
\text { plant }\end{array}$ & $\begin{array}{l}\text { kernels/ } \\
\text { spike }\end{array}$ & $\begin{array}{l}\text { Spike kernel } \\
\text { weight (g) }\end{array}$ & $\begin{array}{l}\text { 100-kernel } \\
\text { weight (g) }\end{array}$ & $\begin{array}{l}\text { Grain } \\
\text { yield / } \\
\text { plant (g) }\end{array}$ \\
\hline $\mathrm{L}_{1}$ & $-3.542^{*}$ & $-0.514^{*}$ & $-10.736^{*}$ & $-0.251^{*}$ & 0.072 & -0.146 \\
\hline $\mathrm{L}_{2}$ & $3.958^{*}$ & $0.486^{*}$ & $5.264^{*}$ & $0.463^{*}$ & $0.317^{*}$ & $4.266^{*}$ \\
\hline $\mathrm{L}_{3}$ & $-2.708^{*}$ & $0.486^{*}$ & 0.264 & $-0.162^{*}$ & 0.016 & $-1.763^{*}$ \\
\hline $\mathrm{L}_{4}$ & $-6.042^{*}$ & $-1.180^{*}$ & $1.014^{*}$ & $-0.277^{*}$ & $-0.361^{*}$ & $-4.349^{*}$ \\
\hline $\mathrm{L}_{5}$ & $2.292^{*}$ & -0.014 & $-5.069^{*}$ & $-0.172^{*}$ & $-0.243^{*}$ & $3.174^{*}$ \\
\hline $\mathrm{L}_{6}$ & $-3.542^{*}$ & $-1.014^{*}$ & $2.681^{*}$ & $0.293^{*}$ & $0.330^{*}$ & $-0.984^{*}$ \\
\hline $\mathrm{L}_{7}$ & $5.625^{*}$ & $-0.180^{*}$ & $5.431^{*}$ & $0.233^{*}$ & $-0.234^{*}$ & $0.933^{*}$ \\
\hline $\mathrm{L}_{8}$ & $3.959^{*}$ & $0.319^{*}$ & $-5.903^{*}$ & $-0.204^{*}$ & $-0.095^{*}$ & $-1.748^{*}$ \\
\hline $\mathrm{L}_{9}$ & $2.292^{*}$ & $0.986^{*}$ & $8.430^{*}$ & $0.287^{*}$ & $-0.197^{*}$ & $3.125^{*}$ \\
\hline $\mathrm{L}_{10}$ & $-4.375^{*}$ & -0.014 & $-3.153^{*}$ & $-0.217^{*}$ & $0.113^{*}$ & $-1.303^{*}$ \\
\hline $\mathrm{L}_{11}$ & $0.625^{*}$ & 0.153 & $-3.903^{*}$ & $-0.127^{*}$ & $0.213^{*}$ & $-3.038^{*}$ \\
\hline $\mathrm{L}_{12}$ & $1.458^{*}$ & $0.486^{*}$ & $5.680^{*}$ & $0.134^{*}$ & 0.069 & $1.833^{*}$ \\
\hline $\mathrm{L}_{\text {L.S.D. 5\% (gi-gj) }}$ & 0.352 & 0.159 & 0.857 & 0.058 & 0.074 & 0.261 \\
\hline Testers & \multicolumn{5}{|l|}{} & $-0.220^{*}$ \\
\hline $\mathrm{T}_{1}$ & $-5.208^{*}$ & $-0.222^{*}$ & $-5.840^{*}$ & -0.028 & $0.181^{*}$ & $-0.318^{*}$ \\
\hline $\mathrm{T}_{2}$ & $0.833^{*}$ & $0.153^{*}$ & $1.555^{*}$ & $-0.261^{*}$ & -0.310 & $0.538^{*}$ \\
\hline $\mathrm{T}_{3}$ & $4.375^{*}$ & 0.069 & $4.285^{*}$ & $0.289^{*}$ & $0.129^{*}$ & 0.130 \\
\hline L.S.D. 5\% (gi-gj) & 0.176 & 0.080 & 0.429 & 0.029 & 0.037 & \\
\hline
\end{tabular}

* Significant at 0.05 level of probability. 


\section{Specific combining ability (SCA)}

Results for specific combining ability effects of the studied characters are presented in Table (5). Significant negative specific combining ability effects for plant height were detected in 17 crosses. Significant positive (SCA) effects for spikes per plant was detected in eleven crosses, for kernels per spike was detected in fourteen crosses, for spike kernel weight was detected in ten crosses, for kernel weight was detected in seven crosses and for grain yield per plant was detected in thirteen crosses. It could be concluded that Sids-12 might be used as parental material for wheat breeding programs since it is considered a common parent in such crosses for kernels per spike, spike kernel weight and grain yield per plant.

The results obtained herein concerning general and specific combining ability effects indicated that excellent hybrid combinations were obtained from crosses of high $x$ high, high $x$ low and low $x$ low GCA effects. It could be concluded that general combining ability effects were generally unrelated to the specific combining ability effects of their respective crosses.

Therefore, from these results it may be concluded that in selection of parents for use in hybrid combinations, it would be more profitable to select first on the basis of their general combining abilities, and further selection might then be based on the specific combining ability effects. This conclusion was previously reported by Moubarak (2003), Koumber and El-Beially (2005), Koumber et. al. (2006), Moshref (2006), Koumber (2007) and Krystkowiak et. al. (2009). 
Table 5. Estimates of specific combining ability (SCA) effects for all the studied characters.

\begin{tabular}{|c|c|c|c|c|c|c|c|}
\hline \multicolumn{2}{|c|}{ Hybrids } & $\begin{array}{l}\text { Plant } \\
\text { height } \\
(\mathrm{cm} .) \\
\end{array}$ & $\begin{array}{l}\text { spikes/ } \\
\text { plant }\end{array}$ & $\begin{array}{l}\text { kernels/ } \\
\text { spike }\end{array}$ & $\begin{array}{l}\text { Spike kernel } \\
\text { weight }(\mathrm{g})\end{array}$ & $\begin{array}{l}100- \\
\text { kernel } \\
\text { weight }(\mathrm{g})\end{array}$ & $\begin{array}{l}\text { Grain } \\
\text { yield / } \\
\text { plant (g) }\end{array}$ \\
\hline \multirow{3}{*}{$\mathrm{L}_{1}$} & $x T_{1}$ & 0.208 & $0.889^{*}$ & $6.174^{*}$ & $0.677^{*}$ & 0.110 & $6.452^{*}$ \\
\hline & $\times T_{2}$ & $1.666^{*}$ & 0.014 & $-7.222^{*}$ & $-0.485^{*}$ & $0.173^{*}$ & $-5.301^{*}$ \\
\hline & $\times T_{3}$ & $-1.875^{*}$ & $-0.902^{*}$ & 1.049 & $-0.192^{*}$ & $-0.283^{*}$ & $-1.151^{*}$ \\
\hline \multirow{3}{*}{$\mathrm{L}_{2}$} & $\times T_{1}$ & 0.208 & $-0.611^{*}$ & $2.174^{*}$ & -0.022 & 0.010 & $-1.610^{*}$ \\
\hline & $x T_{2}$ & $-0.833^{*}$ & $-0.486^{*}$ & $-2.972^{*}$ & -0.036 & $-0.310^{*}$ & $-3.127^{*}$ \\
\hline & $\times T_{3}$ & $0.625^{*}$ & $1.097^{*}$ & 0.798 & 0.058 & $0.299^{*}$ & $4.737^{*}$ \\
\hline \multirow{3}{*}{$\mathrm{L}_{3}$} & $\times \mathrm{T}_{1}$ & $1.875^{*}$ & $-0.611^{*}$ & -1.076 & $0.473^{*}$ & -0.031 & $3.424^{*}$ \\
\hline & $\times T_{2}$ & $-4.167^{*}$ & $-0.486^{*}$ & $5.278^{*}$ & $-0.189^{*}$ & $0.395^{*}$ & $-3.179^{*}$ \\
\hline & $\times T_{3}$ & $2.292^{*}$ & $1.097^{*}$ & $-4.201^{*}$ & $-0.284^{*}$ & $-0.364^{*}$ & -0.245 \\
\hline \multirow{3}{*}{$\mathrm{L}_{4}$} & $\times \mathrm{T}_{1}$ & 0.208 & $1.055^{*}$ & $1.674^{*}$ & -0.068 & $0.139^{*}$ & $1.715^{*}$ \\
\hline & $\times T_{2}$ & $9.167^{*}$ & $-0.319^{*}$ & $4.778^{*}$ & $0.272^{*}$ & -0.091 & 0.040 \\
\hline & $\times T_{3}$ & $-9.375^{*}$ & $-0.736^{*}$ & $-6.451^{*}$ & $-0.203^{*}$ & -0.047 & $-1.756^{*}$ \\
\hline \multirow{3}{*}{$\mathrm{L}_{5}$} & $x T_{1}$ & $1.875^{*}$ & $0.889^{*}$ & $7.757^{*}$ & -0.028 & 0.069 & $4.562^{*}$ \\
\hline & $x T_{2}$ & $-4.167^{*}$ & $-0.486^{*}$ & 1.361 & $0.237^{*}$ & $-0.181^{*}$ & $2.837^{*}$ \\
\hline & $\times T_{3}$ & $2.292^{*}$ & $-0.403^{*}$ & $-9.118^{*}$ & $-0.208^{*}$ & 0.112 & $-7.399^{*}$ \\
\hline \multirow{3}{*}{$\mathrm{L}_{6}$} & $\times T_{1}$ & $-4.791^{*}$ & -0.111 & $-14.993^{*}$ & -0.150 & $-0.142^{*}$ & $-0.839^{*}$ \\
\hline & $\times T_{2}$ & $11.667^{*}$ & $1.514^{*}$ & -0.889 & $-0.419^{*}$ & 0.028 & $4.445^{*}$ \\
\hline & $x T_{3}$ & $-6.875^{*}$ & $-1.403^{*}$ & $15.882^{*}$ & $0.568^{*}$ & 0.114 & $-3.606^{*}$ \\
\hline \multirow{3}{*}{$\mathrm{L}_{7}$} & $\times T_{1}$ & $3.542^{*}$ & $-0.944^{*}$ & $-7.493^{*}$ & -0.065 & -0.038 & $-7.279^{*}$ \\
\hline & $\times T_{2}$ & $-2.500^{*}$ & 0.180 & $-3.889^{*}$ & $-0.129^{*}$ & 0.022 & $1.245^{*}$ \\
\hline & $x T_{3}$ & $-1.042^{*}$ & $0.764^{*}$ & $11.382^{*}$ & $0.193^{*}$ & 0.016 & $6.034^{*}$ \\
\hline \multirow{3}{*}{$\mathrm{L}_{8}$} & $\times T_{1}$ & 0.208 & 0.055 & $-5.410^{*}$ & $-0.755^{*}$ & $-0.227^{*}$ & $-4.608^{*}$ \\
\hline & $\times T_{2}$ & $-5.833^{*}$ & 0.180 & 1.194 & 0.124 & -0.065 & $-1.025^{*}$ \\
\hline & $x T_{3}$ & $5.625^{*}$ & -0.236 & $4.215^{*}$ & $0.631^{*}$ & $0.292^{*}$ & $5.633^{*}$ \\
\hline \multirow{3}{*}{$\mathrm{L}_{9}$} & $\mathrm{xT} \mathrm{T}_{1}$ & $11.875^{*}$ & $0.889^{*}$ & $6.257^{*}$ & -0.043 & $0.252^{*}$ & $-0.531^{*}$ \\
\hline & $x T_{2}$ & $-4.167^{*}$ & $-0.486^{*}$ & -1.389 & $0.160^{*}$ & $-0.213^{*}$ & $2.859^{*}$ \\
\hline & $x T_{3}$ & $-7.708^{*}$ & $-0.403^{*}$ & $-4.868^{*}$ & $-0.117^{*}$ & -0.039 & $-2.327^{*}$ \\
\hline \multirow{3}{*}{$\mathrm{L}_{10}$} & $x T_{1}$ & $-6.458^{*}$ & -0.111 & 0.090 & $-0.143^{*}$ & $-0.153^{*}$ & 0.422 \\
\hline & $x T_{2}$ & $2.500^{*}$ & $0.514^{*}$ & $6.944^{*}$ & $0.309^{*}$ & $0.155^{*}$ & $-0.478^{*}$ \\
\hline & $x T_{3}$ & $3.958^{*}$ & $-0.403^{*}$ & $-7.035^{*}$ & $-0.166^{*}$ & -0.002 & 0.056 \\
\hline \multirow{3}{*}{$\mathrm{L}_{11}$} & $\times T_{1}$ & $-6.458^{*}$ & $-0.778^{*}$ & $10.590^{*}$ & 0.104 & -0.010 & $-1.458^{*}$ \\
\hline & $\times T_{2}$ & $-7.500^{*}$ & $-0.653^{*}$ & $-6.305^{*}$ & $0.177^{*}$ & -0.032 & -0.181 \\
\hline & $x T_{3}$ & $13.958^{*}$ & $1.431^{*}$ & $-4.285^{*}$ & $-0.281^{*}$ & 0.042 & $1.640^{*}$ \\
\hline \multirow{3}{*}{$\mathrm{L}_{12}$} & $\times T_{1}$ & $-2.292^{*}$ & $-0.611^{*}$ & $-5.743^{*}$ & 0.020 & 0.021 & -0.250 \\
\hline & $\times T_{2}$ & $4.167^{*}$ & $0.514^{*}$ & $3.111^{*}$ & -0.021 & 0.119 & $1.865^{*}$ \\
\hline & $\times T_{3}$ & $-1.875^{*}$ & 0.097 & $2.632^{*}$ & 0.001 & $-0.140^{*}$ & $-1.616^{*}$ \\
\hline \multicolumn{2}{|c|}{$\begin{array}{l}\text { L.S.D } 0.05 \text { (Sij- } \\
\text { Sik) }\end{array}$} & 0.609 & 0.276 & 1.485 & 0.101 & 0.127 & 0.452 \\
\hline
\end{tabular}

Significant at 0.05 level. 


\section{REFERENCES}

1. Abd El-Rahman, M.F.S. 1991. Gene action in the inheritance of yield and its components in wheat. M.Sc. Thesis, Fac. Agric. Ain Shams Univ., Egypt.

2. Akbar, M., J. Anwar, M. Hussain, M.H. Qureshi and S. Khan. 2009. Line $x$ tester analysis in bread wheat( Triticum aestivum L.). J. Agric. Res. Lahore, 47:21-30.

3. Chowdhry, M.A., M.T. Mahmood and I. Khaliq. 1996. Genetic analysis of some drought and yield related characters in Pakistan spring wheat varieties. Wheat Information Service, 82:8-11.

4. Eissa, M.M. 1993. Combining ability for main spike characteristics in durum wheat (Triticum turgidum var. durum). Zagazig J. Agric. Res. 20:1673-1681.

5. El-Beially, I.E.M.A., and E.A.M. El-Sayed. 2002. Heterosis and combining ability for some bread wheat crosses. J. Agric. Sci. Mansoura Univ. 27:5735-5744.

6. Kempthorne, O. 1957. An Introduction to Genetic Statistics. p. 475-491. John Wiley-Sons inc. New York.

7. Koumber, R.M.A. 2007. Estimation of combining ability for yield and its components in bread wheat using line $x$ tester analysis. J. Agric. Sci. Mansoura Univ. 32:741-749.

8. Koumber, R.M.A., and E.M.A. El-Beially. 2005. Genetic analysis for yield and its attributes in bread wheat. J. Agric. Sci. Mansoura Univ. 30:1827-1838.

9. Koumber, R.M.A., E.M.A. El-Beially, and G.A. El-Shaarawy. 2006. Study of genetic parameters and path coefficients for some quantitative characters in wheat under two levels of nitrogen fertilizer. Al-Azher J. Agric. Res. Al-Azher Univ., 43: 99-122.

10. Krystkowiak, K., T. Adamski, M. Surma, and Z. Kaczmarek. 2009. Relationship between phenotypic and genetic diversity of parental genotypes and the specific combining ability and heterosis effects in wheat ( Triticum aestivum L.). Euphytica, 165:419-434.

11. Moshref, M.Kh. 2006. Studies of gene effect on yield and yield components for some bread wheat crosses. J. Agric. Sci. Mansoura Univ., 31:3273-3283.

12. Moubarak, M.Y.Gh. 2003. Heterosis and combining ability of some bread wheat germplasm at New Valley region. M.Sc. Thesis, Fac. Agric., Minia Univ., Egypt.

13. Srivastava, M.K., and S. Dhirendra. 2008. Study on heterosis with respect to yield in bread wheat [Triticum aestivum (L.) em Thell]. Research on Crops., 9:151-154. 


\section{القدرة علي الائتلاف لبعض أصناف وسلالات قمح الخبز باستخدام تحليل السلالة × الكشاف تحت ظروف الوادي الجديا}

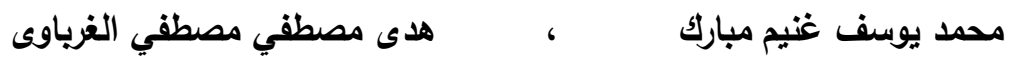

$$
\begin{aligned}
& \text { قسم بحوث القمح - معرة بحوث الدحاصيل الحقلية - مركز البحوث الزراعية - جيزة } \\
& \text { - مصر. }
\end{aligned}
$$

أجريت هذه الدراسة بمحطة البحوث الزراعية بالوادي الجديد ، التابعة لمركز البحوث الزراعية ـ

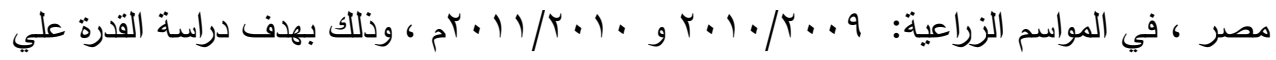
الاتتلاف للصفات التالية : طول النبات ، عدد سنابل النبات ، عدد حبوب السنبلة ، وزن حبوب السنبلة

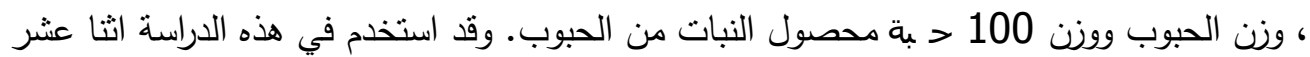

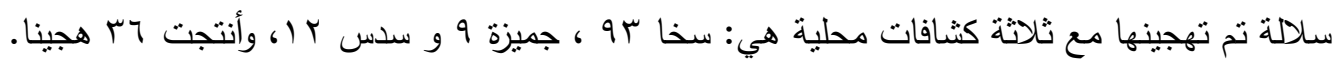

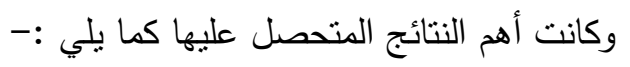

- كانت قيم التباين الراجعة إلي كل من الآباء وتاهجن والآباء مع الهجن معنوية لكل الصفات

$$
\text { تحت الدراسة. }
$$

- أظهرت النتائج أن الفعل الجيني غير المضيف كان الأكثر أهمية في نوارث الصفات تحت الدراسة مقتارنة بالفعل الجيتى المضيف.

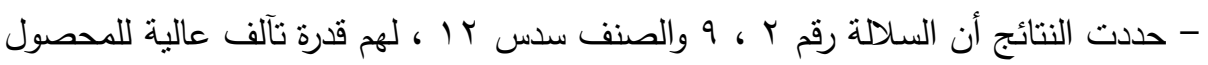
ومكوناته ، ويمكن الاستفادة منها بإدخالها في برامج التربية كمواد وراثية جيدة. 